\title{
Synthesis, characterization and nonlinear optical properties of silver/PVA nanocomposites
}

\section{N. Faraji}

nastaran.faraji@gmail.com

\section{W. Mahmood Mat Yunus}

\section{A. Kharazmi \\ E. Saion}

\section{Shahmiri}

\section{N. Tamchek}

\author{
Department of Physics, Faculty of Science, Universiti Putra Malaysia, 43400 UPM Serdang, Selangor, \\ Malaysia \\ Department of Physics, Faculty of Science, Universiti Putra Malaysia, 43400 UPM Serdang, Selangor, \\ Malaysia \\ Department of Physics, Faculty of Science, Universiti Putra Malaysia, 43400 UPM Serdang, Selangor, \\ Malaysia \\ Department of Physics, Faculty of Science, Universiti Putra Malaysia, 43400 UPM Serdang, Selangor, \\ Malaysia \\ Department of chemistry, Faculty of Science, Universiti Putra Malaysia, 43400 UPM Serdang, Selangor, \\ Malaysia \\ Department of Physics, Faculty of Science, Universiti Putra Malaysia, 43400 UPM Serdang, Selangor, \\ Malaysia
}

Silver/polyvinyl alcohol (PVA) nanocomposites are prepared via quick precipitation method, using hydrazine as a reducing agent. Preparing of silver/PVA nanocomposites by this method is done for the first time. The samples are characterized by Uv-Visible spectroscopy, X-ray diffraction (XRD), transmission electron microscopy (TEM). Nonlinear optical properties are carried out by Z-scan technique using a blue $\mathrm{CW}$ laser beam operated at wavelength $405 \mathrm{~nm}$. It is shown that the synthesized samples have negative nonlinear refractive index and the magnitude is in the order of $10^{-8}$. The nonlinear refractive index increases as amount of reducing agent increases.

[DOI: http://dx.doi.org/10.2971/jeos.2012.12040]

Keywords: Silver nanocomposites, z-scan measurement, nonlinear refractive index, nonlinear absorption coefficient

\section{INTRODUCTION}

Nanoscience and nanotechnology primarily deal with the synthesis, characterization, exploration, and exploitation of nanostructure materials [1] and recently this field has provided solutions to many technological challenges, e.g., solar cell, medicine, water treatment [2]. Nanoscale metals show different properties than their bulk counterparts. Their unique optical, electrical and magnetic properties depend on the shape and size of the nanoparticles [3]. Among these nanometals, silver nanoparticles are particularly important due to their high electrical and thermal conductivities [4].

It has been shown that polymers are suitable host material for metal nanoparticles with known characteristics [5]-[10]. Due to electrical, magnetic and optical properties and the ability to preclude oxidation and agglomeration, polymer based nanocomposites have attracted more attention [7]. There are many reports for the synthesis of metal nanocomposites using different methods, e.g., gamma radiation [9], chemical reduction method [5]-[6], sol-gel method [11], and laser ablation [7]. In the most of the cases the polymer is prepared in the first step, then metal ions are entered into polymers, and ions are reduced to zero valent state by reducing agent or by heating [9].

For metallic nanoparticles such as Ag, several investigations concerning the nonlinear optical response have been done and until the commencement of the laser as a source of photon, most of the optical nonlinear responses were not exhibited [12]. Furthermore the optical nonlinearities of such media are attractive for applications in optical limiting, optical devices, and optical signal processing [13]. Optical nonlinearities are used in optical phase conjugation that is a research topic the past few years [14]. For this propose Z-scan is a simple method for measuring both nonlinear refraction index and nonlinear refractive absorption. This method has been used to determine the sign of nonlinear refractive index too [15]. A sample is scanned through the minimum waist point of the Gaussian laser beam and the far field transmittance is measured after an aperture [16]. Depending on the sign of nonlinear refractive index and the position of the sample relative to the focus point, the sample produces both focusing and defocusing pattern in the far-field [16]. While there are many reports that study the nonlinear optical response of metal colloid under pulsed laser excitation, there have been some studies that have investigated CW laser excitation [17]-[19]. Ganeev et al. reported the nonlinear optical parameters of metal colloidal solutions using Z-scan method using $532 \mathrm{~nm}, 28 \mathrm{~ns}$ and $1064 \mathrm{~nm}, 35 \mathrm{ps}$ laser pulses [13]. Jia et al. used the Zscan technique to investigate the nonlinear optical properties of gold and silver colloidal solution under CW $632 \mathrm{~nm}$ ex- 
citation [17]. In [19] there is a thorough review on nonlinear optical mechanisms of the inorganic nanostructures for optical limiting applications. It summarizes different experiment methods used in research community.

In this paper we present our synthesis method, structural and nonlinear optical properties of silver nanocomposites which are produced, to the best of our knowledge, for the first time by quick precipitation method in PVA liquid. We study the nonlinear refraction and nonlinear absorption of Ag nanocomposites made by different amounts of reducing agent under CW laser excitation which is operated on $405 \mathrm{~nm}$ wave length. In our work the nonlinear optical properties of silver/PVA nanocomposites is caused by laser heating.

\section{EXPERIMENTAL WORKS}

\subsection{Materials}

Silver nitrite $\left(\mathrm{AgNO}_{3}\right)$ was obtained from MERK and polyvinyl alcohol (PVA) with the weight average number of 88000 was obtained from ACROS. Hydrazine $\left(\mathrm{N}_{2} \mathrm{H}_{4}\right)$ was purchased from ACROS which has been used as a reducing agent.

\subsection{Synthesis of silver nanocomposites}

The silver nanocomposites are synthesized by following steps. $5 \mathrm{wt} \%$ PVA solution is prepared by dissolving PVA powder in deionized water at the $90^{\circ} \mathrm{C}$ in water bath. After cooling down PVA solution to the room temperature, it was divided equally into 5 bottom flasks. Silver nitrite was added to PVA at concentration of $0.1 \mathrm{M}$. To prevent samples from oxidation they were bubbled by nitrogen gas for 5 minutes and then continuously stirred for 10 minutes. $1 \mathrm{M}$ ratio of $\mathrm{N}_{2} \mathrm{H}_{4}$ as reducing agent was added suddenly with the amount of $1,2,5,10$, and $15 \mathrm{ml}$. Finally samples were stirred in water bath for an hour at $60^{\circ} \mathrm{C}$.

\subsection{Characterization}

The optical spectra of silver nanocomposites in PVA were record by UV-3600, Shimadzu spectrophotometer. X-ray diffraction measurement was done by XRD 6000 Shimadzu diffractometer and by using $\mathrm{Cuk}_{\alpha}(0.154 \mathrm{~nm})$ radiation at the room temperature. The average size of the nanocomposites was recorded by transmission electron spectroscopy (JOEL $2010 \mathrm{~F}$ UHR) operated at $200 \mathrm{kV}$. For preparation the sample was diluted in water and after 10 minutes ultrasonic, a drop of sample was put on the copper grid and samples were dried and kept under vacuum in the desiccators.

\subsection{Nonlinear optical properties measurement}

The nonlinear properties of silver nanocomposites were investigated by using simple Z-scan technique. Figure 1 shows schematic diagram of experimental Z-scan experiment which was use in our measurement. The wavelength of the laser used in the experiments was $405 \mathrm{~nm}$. The sample was placed

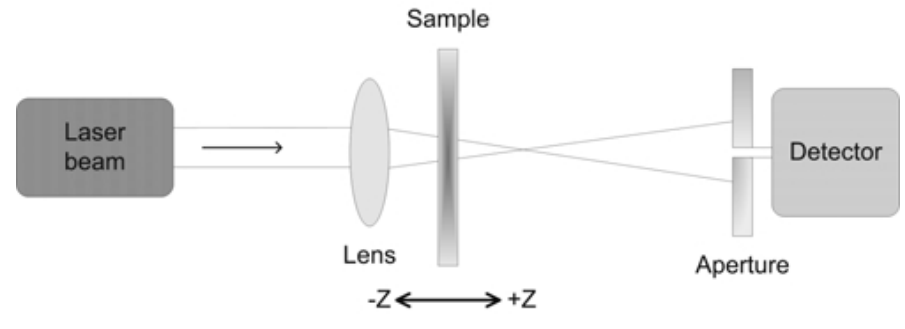

FIG. 1 Schematic diagram of Z-scan measurement setup for close aperture.

in a quartz cell with a thickness of $1 \mathrm{~mm}$ for the measurement. The quartz cell was moved across the focal point of the lens by using BSC101 micro stepping controller from Thorlabs that was controlled by Lab View. The focal length of lens was $10 \mathrm{~cm}$ which focused the Gaussian beam to a spot size with a radius of $\sim 42 \mu \mathrm{m}$ at the beam waist. The input and output powers were measured by $1936-\mathrm{C}$ power meter from Newport. The detected power has been normalized by the power value observed when the quartz cell located at the focal point of the lens [20]. In our measurement the sample could be considered as a thin medium due to the satisfied the condition $\pi \omega_{0}^{2} / \lambda>L$, where $\omega_{0}$ is beam waist at focal point, $L$ is the thickness of the sample and $\lambda$ is the wavelength of the beam. An aperture placed before the detector (close aperture mode) was used to disassociate the nonlinear refractive from the nonlinear absorption, which in second one we omit the aperture or enlarge it (open aperture).

\section{RESULTS AND DISCUSSION}

By increasing the $\mathrm{N}_{2} \mathrm{H}_{4}$ as a reducing agent, the concentration of nanocomposites and the size of the particles increase. It was observed that the colloids could be preserved for more than a month without any obvious changes in color and without any agglomeration being formed. The characterization and nonlinear properties are discussed in this section.

\subsection{Characterization of silver nanocomposites}

Figure 2 shows the XRD patterns of the silver nanoparticles in PVA. It can be seen clearly that the samples consist of crystalline phases presented by five main diffraction peaks at $2 \theta=38.117^{\circ}, 44.301^{\circ}, 64.447^{\circ}, 77.402^{\circ}$, and $81.545^{\circ}$ that correspond to the lattice planes (111), (002), (022), (113), and (222). The broad peak presented by peak $2 \theta=19.19^{\circ}$ is due to PVA existence [21]. In addition, the XRD patterns show that the crystal structure of the sample is cubic. Synthesis of silver nanoparticles in PVA was confirmed by JCPDS 98-006-2694. As the amount of reducing agent increases in the samples, XRD patterns show decreasing in impurities of PVA phase and increasing in crystalline phases, which in the solution with $15 \mathrm{ml}$ of $\mathrm{N}_{2} \mathrm{H}_{4}$ we can clearly see the peaks are wide and grown that can be the prove of increasing the crystalline phases. There is no diffraction peak for $\mathrm{Ag}_{2} \mathrm{O}$ suggesting that our bubblezation during the synthesis prevents the $\mathrm{Ag}$ core from oxidation during synthesis.

Silver nanoparticles absorption characteristic was studied by 


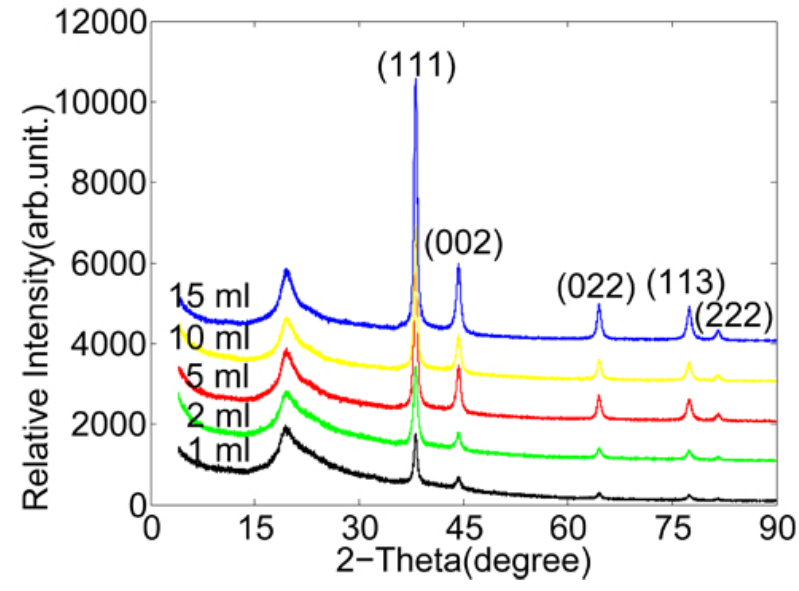

FIG. 2 The X-ray diffraction pattern of silver nanoparticles in PVA using Hydrazine as a reducing agent.

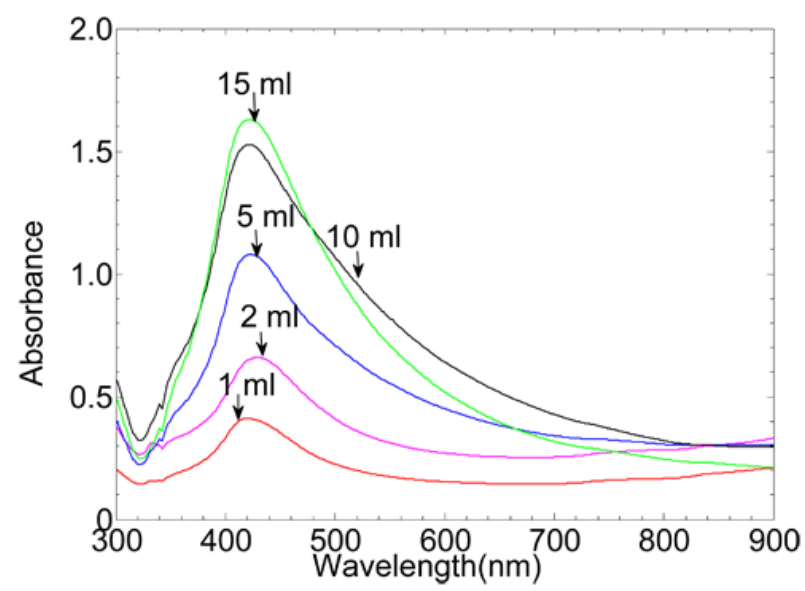

FIC. 3 UV-Vis absorption spectra of silver nano-particles prepared in PVA with various amounts of reducing agent.

UV-Vis spectroscopy. Figure 3 shows the colloidal silver UVVis spectra. In most experiments silver nanoparticles show surface plasmon resonance in the interval 400-430 nm [22]. Here silver plasmon resonance appears at $419 \mathrm{~nm}$ and it goes slightly to higher wavelength (red shift) by increasing the amount of reducing agent. This shift to higher wavelengths also shows an increase in the size due to capping agent. In addition, this increase in size was observed by transmission electron spectroscopy (TEM). Figure 4 shows typical TEM images of silver nanocomposites and their size distribution. It can be seen clearly that they are spherical in shape and no agglomeration is observed in the images. The particles sizes obtained by TEM on average are in 6 to $25 \mathrm{~nm}$ range, which increase as the reducing agent increases in solution.

\subsection{Nonlinear properties of silver colloids}

In this part we present the nonlinear optical properties of silver nanocomposites. In general the nonlinear refraction and nonlinear absorption in the presence of high intensity laser can be described in terms of nonlinear refractive index $n_{2}$ and nonlinear absorption coefficient $\beta$ through:

$$
n(I)=n_{0}+n_{2} I,
$$
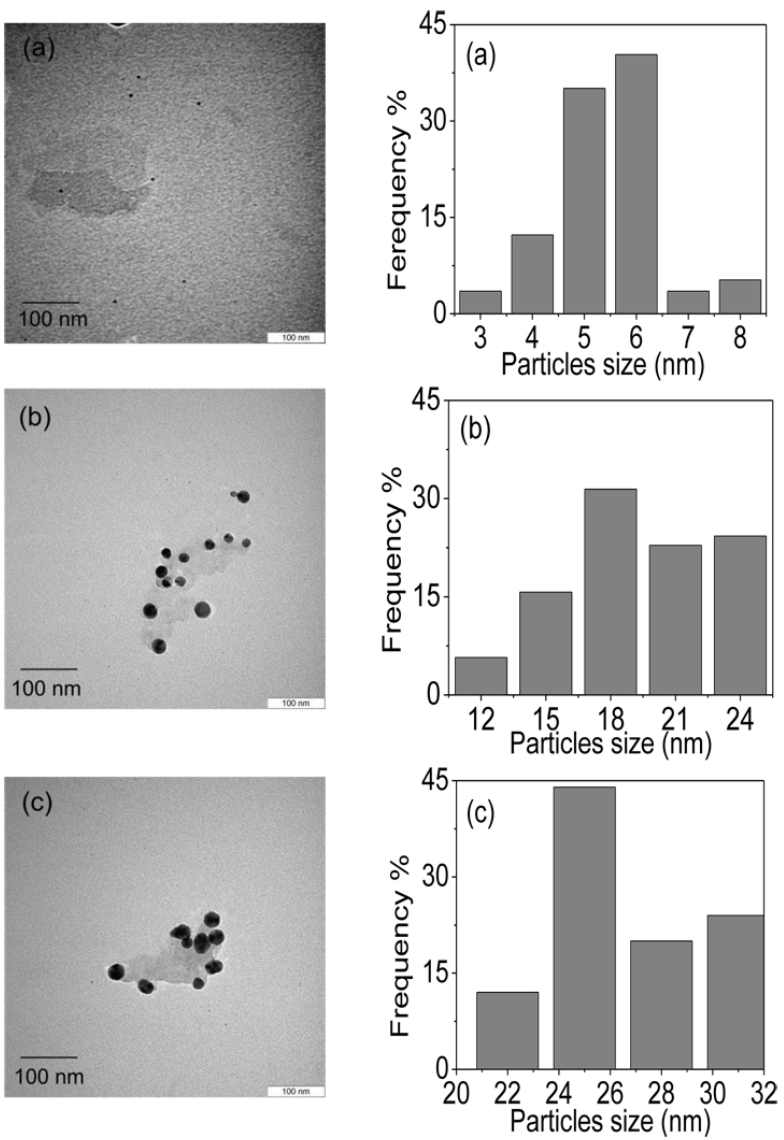

FIG. 4 TEM images and size distributions of silver nanoparticles in PVA; a) for sample with $2 \mathrm{ml}, \mathrm{b}) 5 \mathrm{ml}$, and c) $15 \mathrm{ml}$ reducing agent.

$$
\alpha(I)=\alpha_{0}+\beta I,
$$

where $n_{0}$ is linear refractive index, $\alpha_{0}$ is linear absorption coefficient and $I$ is laser intensity.

The Z-scan curves for close and open aperture for silver nanocomposites prepared by chemical method with different amount of reagent $1,2,5,10$ and $15 \mathrm{ml}$ respectively are shown in Figures 5 and 7. The circles represent the experimental data and solid lines are the theoretical fits. In the first series of experiment, the aperture was placed in front of the detector and it was partially open. Transmittance is related to the nonlinear refractive index by following equation [23]:

$$
\Delta T(z)=1+\frac{4 \Delta \phi_{0} x}{\left(x^{2}+1\right)\left(x^{2}+9\right)}
$$

Where $x$ is normalized distance and it is related to the movement distance of sample $(z)$ through $x=z / z_{0}$ and $z_{0}$ is the Rayleigh length and $\Delta \phi_{0}$ is the phase change. The nonlinear refractive index $n_{2}$ is related to phase change by $n_{2}=\Delta \phi_{0} / k L_{e f f} I_{0}$, where $k=2 \pi / \lambda$ is the wave vector, $L_{e f f}=1-\exp \left(-\alpha_{0} L\right) / \alpha_{0}$ is the effective length of nonlinear medium, $I_{0}$ is on-axis irradiance at focus, $\alpha_{0}$ is the linear absorption coefficient of the samples and $L$ is the sample thickness. The quartz cell thickness was $1 \mathrm{~mm}$ in the experiment. The theoretical transmittance curves fit well to the experimental data. We noticed at $\Delta \phi_{0}=-1.1,-1.32,-1.88,-2.9$, and -3.36 respectively for samples from 1 to $15 \mathrm{ml}$ containing 

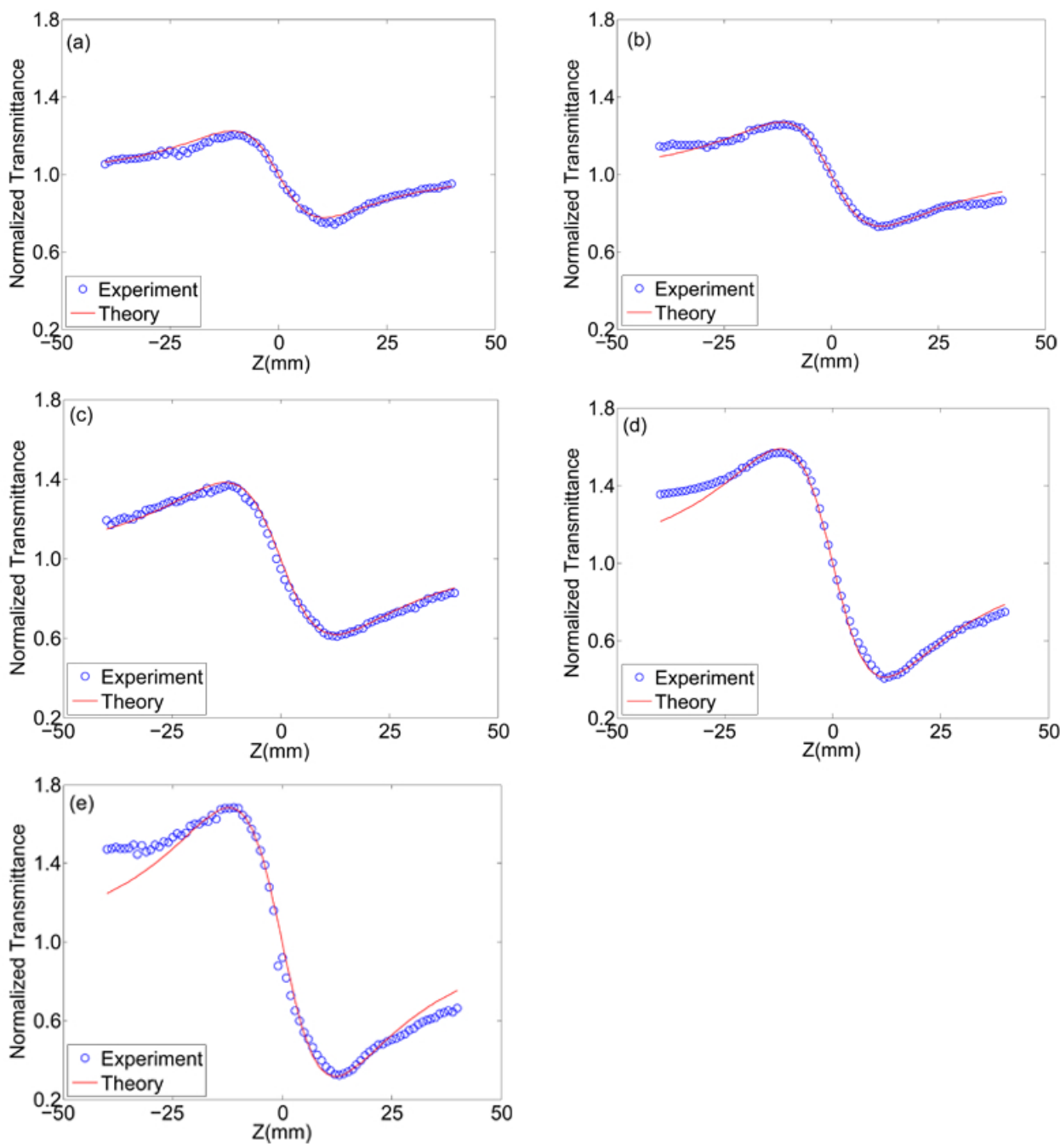

FIG. 5 Normalized Z-scan curves (close aperture) of silver nanoparticles in PVA, using $405 \mathrm{~nm}$ laser. a) $1 \mathrm{ml}$ of $\mathrm{N}_{2} \mathrm{H}_{4}$ b) $2 \mathrm{ml}$ of $\mathrm{N}_{2} \mathrm{H}_{4} \mathrm{C}$ ) $5 \mathrm{ml}$ of $\mathrm{N}_{2} \mathrm{H}_{4} \mathrm{~d}$ ) $10 \mathrm{ml}$ of $\mathrm{N}_{2} \mathrm{H}_{4}$ e) $15 \mathrm{ml}$ of $\mathrm{N}_{2} \mathrm{H}_{4}$. The solid curve shows the model results.
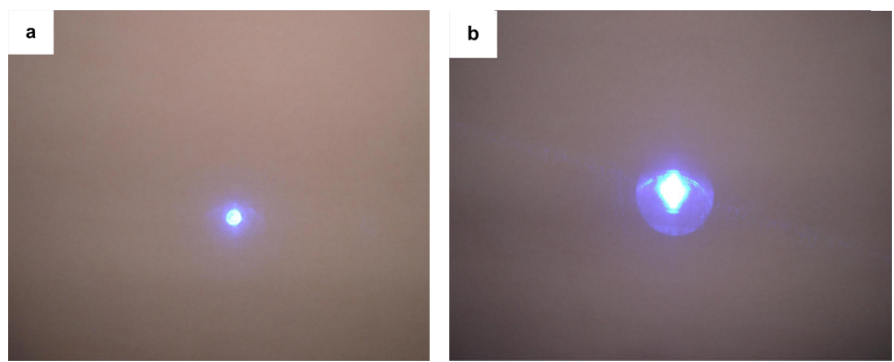

FIC. 6 Far field laser beam profile: a) before the focal point of the lens, b) after focal point.

reducing agent at the best correlation between the experimental data and theoretical transmittance curves observed. The value of nonlinear refractive index $n_{2}$ calculated and it was found to be negative which means the sample can be considered as a thin negative lens. We start the scanning by moving the sample along z-axis, before the focal point of the laser beam was collimated that causes an increase in the transmittance. After the focal point the negative lens diverge the laser beam and causes decreasing irradiance in the far field pattern. A pre-focus maximum followed by a post-focus minimum indicates that the induced lens in negative [24, 25]. Figure 6 shows the picture of the laser beam profile before and after the lens focal point, where the transmittance increase and decrease respectively.

In the second series of experiments an open aperture was inserted before the detector thus the measurements were carried out to measure the nonlinear absorption. In this case we enlarged the aperture to lead the entire beam to the detector. Figure 7 shows all samples have two photon absorption due to their valley structured of the open aperture results. Transmittance beam can be described by [15]:

$$
T(z)=\frac{\ln \left[1+q_{0}(z)\right]}{q_{0}(z)} \text { for }\left|q_{0}(z)\right|\langle 1
$$

where $q_{0}(z)=I_{0} \beta L_{e f f} /\left(1+z^{2} / z_{0}^{2}\right)$ and $\beta$ is the nonlinear absorption coefficient. There is a good agreement between the experimental data and theoretical model fit based on Eq. (4).

The Z-scan measurement was carried out for all five samples and the results are listed in the Table 1 . The nonlinear refractive index $n_{2}$ (close aperture scanned) and nonlinear absorption coefficient $\beta$ (open aperture scanned) respectively refer to real part and imaginary parts of the third order nonlinear susceptibility through [26]:

$$
\chi^{(3)}=\chi_{r}^{(3)}+i \chi_{i}^{(3)}
$$



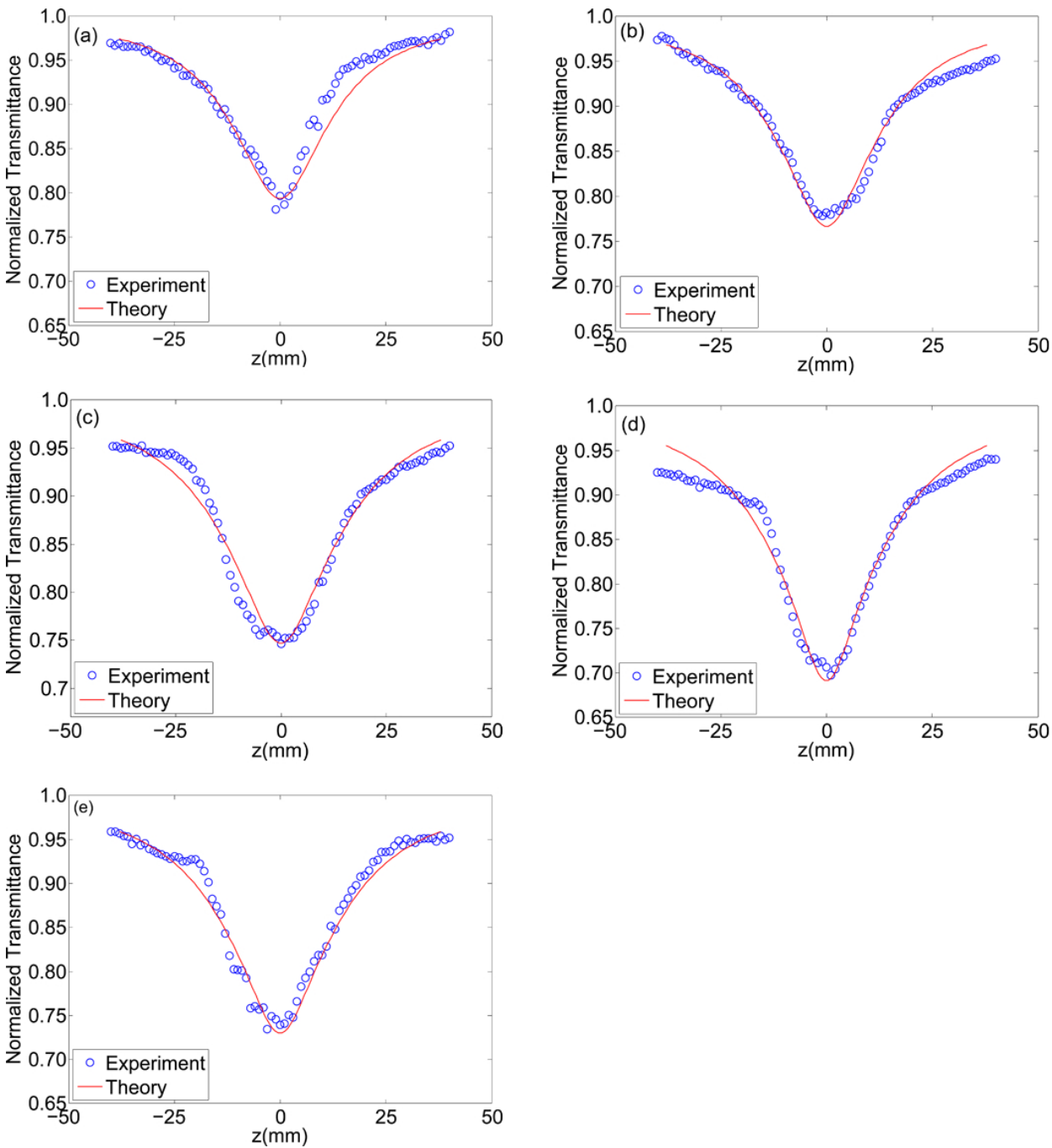

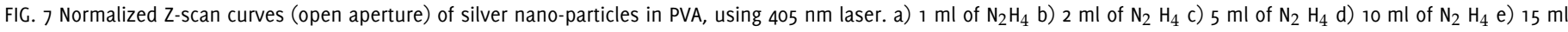
of $\mathrm{N}_{2} \mathrm{H}_{4}$. The solid curve shows the model results.

$$
\begin{aligned}
& \operatorname{Re} \chi^{(3)}(e s u)=10^{-4} \frac{\varepsilon_{0} c^{2} n_{0}^{2}}{\pi} n_{2}\left(\mathrm{~cm}^{2} \mathrm{~W}^{-1}\right), \\
& \operatorname{Im} \chi^{(3)}(e s u)=10^{-2} \frac{\varepsilon_{0} c^{2} n_{0}^{2} \lambda}{4 \pi^{2}} \beta\left(\mathrm{cmW}^{-1}\right) .
\end{aligned}
$$

The absolute value of third order nonlinear refractive index is calculated by:

$$
\left|\chi^{(3)}\right|=\left[\left(\operatorname{Re} \chi^{(3)}\right)^{2}+\left(\operatorname{Im} \chi^{(3)}\right)^{2}\right]^{1 / 2},
$$

where $n_{0}$ is the linear index of refraction, $c$ is the speed of light in vacuum, and $\varepsilon_{0}$ is the free space permittivity [15].

Various physical mechanisms independently contribute to optical nonlinearities of the materials. In general, there are intensive studies on electronic and non-electronic nonlinearities of the materials. The electronic nonlinearity is very important as it appears in most of the dielectric materials, while nonelectronic nonlinearities response is nonradiative interactions like the change in density and temperature [27]. In our work the nonlinear optical properties of silver/PVA nanocomposites could be caused by laser heating.

These signify temporal variation of optical parameters, particularly refractive index. The laser heating generates acoustic waves which change the density of the sample. This is a slow process in order of few nanoseconds and these phenomena can be observed using CW laser, long laser pulses, or high pulse repetition rate in which increasing heat have a prominent role. In addition, due to increasing the linear absorbance of samples by increasing the reducing agent the nonlinear refractive index increase as it can be seen from Figure 5. Increasing the linear absorbance show increasing in size or distribution. In our experiments and from TEM images it is clear that the size of nano-composites increases. This means that the sample can accumulate more heat by increasing the size or distribution and we can see increasing in nonlinear refractive index.

Origins of asymmetries that are seen in the curves in the Figure 7 are from impurities in the samples. The samples were in liquid state and in addition to the silver nanoparticles there are other materials (materials which are consequence of chemical compounds between the sources) that create impurities. Experiments were done by using CW laser and as it was stated above laser heating creates acoustic waves which move the samples inside the quarts cell. If the samples contain impurities with movements the asymmetric curves will be obtained [28].

From our experiment result, Figure 8 shows the nonlinear re- 


\begin{tabular}{|l|l|l|l|l|l|l|l|l|}
\hline $\begin{array}{l}\text { Ag/PVA } \\
\text { samples }\end{array}$ & $\begin{array}{l}\text { Amount of } \\
\text { reducing } \\
\text { agent }(\mathrm{ml})\end{array}$ & $\Delta \phi_{0}$ & $\alpha_{0}\left(\mathrm{~cm}^{-1}\right)$ & $n_{2}\left(\mathrm{~cm}^{2} W^{-1}\right)$ & $\operatorname{Re} \chi^{(3)}$ & $\beta\left(\mathrm{cm} W^{-1}\right)$ & $\operatorname{Im} \chi^{(3)}$ & $\left|\chi^{(3)}\right|$ \\
\hline S1 & 1 & -1.1 & 0.3654 & $-0.913 \times 10^{-8}$ & $-4.290 \times 10^{-7}$ & $1 \times 10^{-4}$ & $1.514 \times 10^{-8}$ & $4.29310^{-7}$ \\
\hline S2 & 2 & -1.32 & 0.5531 & $-1.158 \times 10^{-8}$ & $-5.458 \times 10^{-7}$ & $1.2 \times 10^{-4}$ & $1.822 \times 10^{-8}$ & $5.461 \times 10^{-7}$ \\
\hline S3 & 5 & -1.88 & 0.9540 & $-1.911 \times 10^{-8}$ & $-9.116 \times 10^{-7}$ & $1.5 \times 10^{-4}$ & $2.305 \times 10^{-8}$ & $9.119 \times 10^{-7}$ \\
\hline S4 & 10 & -2.9 & 1.4094 & $-2.895 \times 10^{-8}$ & $-1.384 \times 10^{-6}$ & $1.71 \times 10^{-4}$ & $2.635 \times 10^{-8}$ & $1.384 \times 10^{-6}$ \\
\hline S5 & 15 & -3.36 & 1.5004 & $-3.369 \times 10^{-8}$ & $-1.622 \times 10^{-6}$ & $1.56 \times 10^{-4}$ & $2.421 \times 10^{-8}$ & $1.623 \times 10^{-6}$ \\
\hline
\end{tabular}

TABLE 1 Values of $\mathrm{n}_{2}, \beta$ and $\chi^{(3)}$ measured for silver/PVA nanocomposites at different amount of using reducing agent.

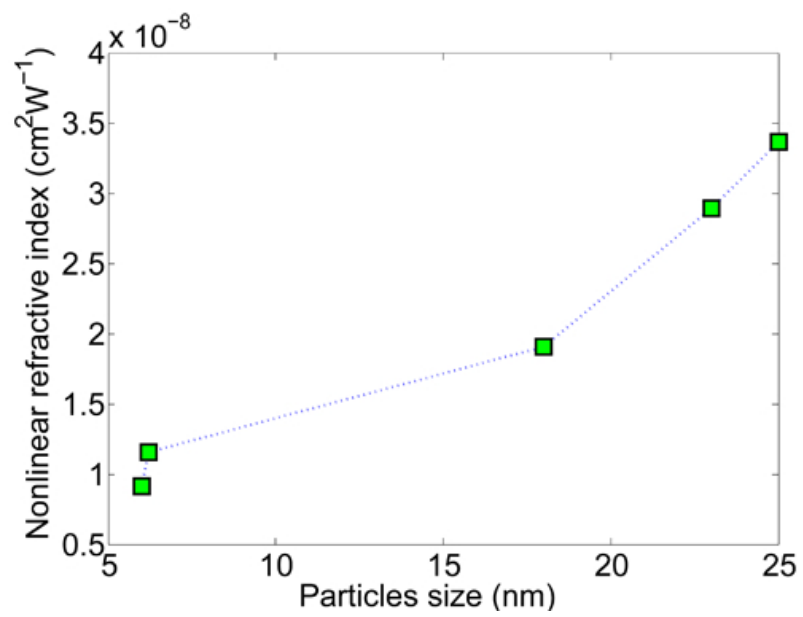

FIG. 8 Nonlinear refractive index as a function of particle size of silver/PVA nanocomposites.

fractive index as a function of particles size, it clearly indicates the increase of the nonlinear refractive index as the particles size increases.

\section{CONCLUSION}

Silver/PVA nanocomposites have been synthesized and their characterization and nonlinear optical properties were studied. The silver nanocomposites are nearly spherical and the size is change from 10 to $25 \mathrm{~nm}$. With simple and sensitive Z-scan technique, the nonlinear refractive index and nonlinear absorption were measured and the sign of nonlinear refractive index was found to be negative. Increase in reducing agent from $1 \mathrm{ml}$ to $15 \mathrm{ml}$, the nonlinear refractive index increase from $-0.913 \times 10^{-8} \mathrm{~cm}^{2} \mathrm{~W}^{-1}$ to $-3.369 \times 10^{-8} \mathrm{~cm}^{2} \mathrm{~W}^{-1}$.

\section{References}

[1] I. U. G. Cogotsi, Nanomaterials handbook (CRC/Taylor at Francis, Boca Raton, 2006).

[2] V. K. Sharma, R. A. Yngard, and Y. Lin, "Silver nanoparticles: Green synthesis and their antimicrobial activities," Adv. Colloid. Interfac. 145, 83-96 (2009).

[3] Z. S. Pillai, and P. V. Kamat, "What Factors Control the Size and Shape of Silver Nanoparticles in the Citrate Ion Reduction Method?" J. Phys. Chem. B 108, 945-951 (2003).

[4] Y. Sun, and Y. Xia, "Large-Scale Synthesis of Uniform Silver Nanowires Through a Soft, Self-Seeding, Polyol Process," Adv.
Mater. 14, 833-837 (2002).

[5] P. K. Khanna, N. Singh, S. Charan, V. V. V. S. Subbarao, R. Gokhale, and U. P. Mulik, "Synthesis and characterization of Ag/PVA nanocomposite by chemical reduction method," Mater. Chem. Phys. 93, 117-121 (2005).

[6] Z. H. Mbhele, M. G. Salemane, C. C. C. E. van Sittert, J. M. Nedeljković, V. Djoković, and A. S. Luyt, "Fabrication and Characterization of Silver-Polyvinyl Alcohol Nanocomposites," Chem. Mater. 15, 5019-5024 (2003).

[7] R. Zeng, M. Z. Rong, M. Q. Zhang, H. C. Liang, and H. M. Zeng, "Laser ablation of polymer-based silver nanocomposites," Appl. Surf. Sci. 187, 239-247 (2002).

[8] Z. Zhang, and M. Han, “One-step preparation of size-selected and well-dispersed silver nanocrystals in polyacrylonitrile by simultaneous reduction and polymerization," J. Mater. Chem. 13, 641-643 (2003).

[9] H. Liu, X. Ge, Y. Zhu, X. Xu, Z. Zhang, and M. Zhang, "Synthesis and characterization of polyacrylamide-nickel amorphous nanocomposites by gamma-irradiation," Mater. Lett. 46, 205-208 (2000).

[10] A. V. Firth, S. W. Haggata, P. K. Khanna, S. J. Williams, J. W. Allen, S. W. Magennis, I. D. W. Samuel, and D. J. Cole-Hamilton, "Production and luminescent properties of CdSe and CdS nanoparticlepolymer composites," J. Lumin. 109, 163-172 (2004).

[11] J. Bai, Y. Li, L. Sun, C. Zhang, and Q. Yang, "Bicomponent AgCl/PVP nanofibre fabricated by electrospinning with gel-sol method," B. Mater. Sci. 32, 161-164 (2009).

[12] D. F. Eaton, "Nonlinear Optical Materials," Science 253, 281-287 (1991).

[13] R. A. Ganeev, A. I. Ryasnyansky, S. R. Kamalov, M. K. Kodirov, and T. Usmanov, "Nonlinear susceptibilities, absorption coefficients and refractive indices of colloidal metals," J. Phys. D Appl. Phys. 34, 1602 (2001).

[14] H. Fei, Z. Wei, Q. Yang, Y. Che, Y. Shen, X. Fu, and L. Qiu, "Lowpower phase conjugation in push pull azobenzene compounds," Opt. Lett. 20, 1518-1520 (1995).

[15] M. Sheik-Bahae, A. A. Said, T. H. Wei, D. J. Hagan, and E. W. Van Stryland, "Sensitive measurement of optical nonlinearities using a single beam," IEEE J. Quantum Elect. 26, 760-769 (1990).

[16] R. L. Sutherland, "Effects of multiple internal sample reflections on nonlinear refractive Z-scan measurements," Appl. Optics 33, 5576-5584 (1994).

[17] T. Jia, T. He, P. Li, Y. Mo, and Y. Cui, "A study of the thermalinduced nonlinearity of $\mathrm{Au}$ and Ag colloids prepared by the chemical reaction method," Opt. Laser Technol. 40, 936-940 (2008).

[18] K. Sendhil, C. Vijayan, and M. P. Kothiyal, "Low-threshold optical power limiting of $\mathrm{cw}$ laser illumination based on nonlinear refrac- 
tion in zinc tetraphenyl porphyrin," Opt. Laser Technol. 38, 512-515 (2006).

[19] J. Wang, and W. J. Blau, "Inorganic and hybrid nanostructures for optical limiting," J. Opt. A-Pure Appl. 0p. 11, 024001 (2009).

[20] Q. W. Song, C. Zhang, R. Gross, and R. Birge, "Optical limiting by chemically enhanced bacteriorhodopsin films," Opt. Lett. 18, 775-777 (1993).

[21] H.M. Zidan, "Effect of $\mathrm{AgNO}_{3}$ filling and UV-irradiation on the structure and morphology of PVA films," Polym. Test. 18, 449-461 (1999).

[22] R. A. Ganeev, M. Baba, A. I. Ryasnyansky, M. Suzuki, and H. Kuroda, "Characterization of optical and nonlinear optical properties of silver nanoparticles prepared by laser ablation in various liquids," Opt. Commun. 240, 437-448 (2004).

[23] G. V. Prakash, M. Cazzanelli, Z. Gaburro, L. Pavesi, F. Iacona, G. Franzo, and F. Priolo, "Nonlinear optical properties of silicon nanocrystals grown by plasma-enhanced chemical vapor deposition," J. Appl. Phys. 91, 4607-4610 (2002).
[24] H. Manaa, A. Tuhl, J. Samuel, A. Al-Mulla, N. A. Al-Awadi, and S. Makhseed, "Photophysical and nonlinear optical properties of zincphthalocyanines with peripheral substitutions," Opt. Commun. 284, 450-454 (2011).

[25] Q. W. Song, C. Zhang, R. B. Gross, and R. R. Birge, "The intensity-dependent refractive index of chemically enhanced bacteriorhodopsin," Opt. Commun. 112, 296-301 (1994).

[26] S. J. Mathews, S. C. Kumar, L. Giribabu, and S. V. Rao, "Large third-order optical nonlinearity and optical limiting in symmetric and unsymmetrical phthalocyanines studied using Z-scan," Opt. Commun. 280, 206-212 (2007).

[27] T. He, and C. Wang, "The study on the nonlinear optical response of Sudan I," Opt. Commun. 281, 4121-4125 (2008).

[28] E. Shahriari, W. M. Mat Yunus, K. Naghavi, and Z. A. Talib, "Effect of concentration and particle size on nonlinearity of Au nano-fluid prepared by $\gamma(60 \mathrm{Co})$ radiation," Opt. Commun. 283, 1929-1932 (2010). 